\title{
Contaminación Atmosférica, Morbilidad y Mortalidad en la ciudad de Albacete (Año 2005)
}

\author{
Ma Jesús López Gigosos ${ }^{a}$
}

\begin{abstract}
a Catedrática de Biología y Geología del Instituto de Enseñanza Secundaria Federico García Lorca (Albacete).

\section{Correspondencia:}

Ma Jesús López Gigosos,

IES Federico García Lorca,

C/ Pintor Quijada n 1 , 02006 - Albacete.

Telf.: 967505130,

correo electrónico:

marilogigosa1@msn.com.
\end{abstract}

Recibido el 9 de septiembre de 2009.

Aceptado para su publicación el 23 de septiembre de 2009.

\begin{abstract}
RESUMEN
Contaminación atmosférica, morbilidad y mortalidad en la ciudad de albacete (año 2005)

Objetivo. Estudiar el efecto a corto plazo de la contaminación atmosférica sobre los ingresos hospitalarios por enfermedades respiratorias, infecciosas, circulatorias, cardiovasculares y sobre la mortalidad general.

Diseño. Estudio transversal ecológico utilizando fuentes de datos diferentes.

Emplazamiento. Albacete capital y año 2005.

Participantes. La población residente en Albacete capital.

Mediciones principales. Variables ambientales (contaminantes y meteorológicas) y variables sanitarias (de morbilidad y mortalidad)

Resultados. No se observan unos altos niveles de contaminación. Los niveles de partículas son altos a finales del invierno, volviendo a ascender en menor medida en verano. Se observó el patrón típico del ozono (O3) en verano y de los óxidos en invierno. Se demuestra una correlación positiva entre los ingresos hospitalarios especialmente por patología respiratoria y los niveles de Óxidos de Nitrógeno (NOx) y Óxidos de Azufre (SOx) en el total de ingresos y en el grupo de 65 o más edad. Así mismo se ha establecido una correlación negativa entre los ingresos hospitalarios por patología respiratoria y los niveles de ozono y temperatura con respecto a los grupos de todas las edades, en el de 65 o más edad y en el de 0-14 años. Los análisis realizados mediante modelos de series temporales ponen de manifiesto la influencia de las variables ambientales estudiadas en la morbilidad, especialmente la de tipo respiratoria, en los mayores de 64 años y en los menores de 15 años.

Conclusiones. A pesar del bajo nivel de contaminación, se observa una relación significativa entre algunos contaminantes atmosféricos y la morbilidad hospitalaria y la mortalidad. En la mayoría de los análisis, el efecto de la contaminación atmosférica es superior en personas mayores de 64 años y en los menores de 15 años. En los análisis de series temporales se observa una significativa implicación de los factores ambientales en la morbilidad por todas las causas, y en particular por causa respiratoria y patología del aparato circulatorio.
\end{abstract}

Palabras clave. Contaminantes del aire. Morbilidad. Mortalidad.

\section{ABSTRACT}

Atmospheric pollution, morbidity and mortality in the city of Albacete (year 2005).

Objective. To study the short term affect of atmospheric pollution on hospital admittances for respiratory, infectious, circulatory and cardiovascular diseases and on general mortality.

Design. Cross sectional, ecological study using different data sources.

Setting. The city of Albacete in 2005.

Participants. The population residing in the city of Albacete.

Main measurements. Environmental variables (pollutants and meteorological factors)

Results. High levels of air pollution were not observed. The level of particulate matter is high at the end of winter and also increases, but to a lesser extent, in summer. The typical patterns for ozone $\left(\mathrm{O}^{3}\right)$ in summer and oxides in winter were observed. In total admittances and in the 65 years or over group, a positive correlation was found between hospital admittances for respiratory diseases and levels of Nitrogen Oxides (NOx) and Sulphur Oxides (SOx). Likewise negative correlations between hospital admittances for respiratory disease and ozone levels and temperature were established for the all ages group, for the 65 years or over group and for the 0-14 years group. The analyses performed by time series models demonstrate that environmental variables have an effect on morbidity, especially respiratory problems, in persons over 64 years and in those under 15 years.

Conclusions. Although there was a low level of air pollution, we found a significant correlation between some air pollutants and hospital morbidity and mortality. In most of the analyses, the effect of air pollution is greater in persons over 64 years and in those under 15 years. The time series analyses revealed that environmental factors had a significant impact on morbidity for all causes, and in particular for respiratory and circulatory system diseases.

Key words. Air Pollutants, Morbidity, Mortality. 


\section{INTRODUCCIÓN}

La contaminación atmosférica y sus efectos, a corto plazo, sobre la salud preocupan desde hace tiempo. Durante la primera mitad del siglo XX tuvieron lugar una serie de episodios en países industrializados que fueron un detonante para el estudio de las relaciones entre contaminación atmosférica y salud humana. Es el caso del Valle de Mosa1 (Bélgica) en 1930, Donora (Pennsylvania, USA) en 1948 (Shrenk, 1949), y especialmente la catástrofe de Londres en diciembre de 1952 (Ministry of Health, 1954). Todos estos hechos catalizaron el desarrollo de políticas de control de la contaminación en Europa Occidental y en Estados Unidos que han traído como consecuencia una reducción de los niveles de contaminación y por tanto de la morbilidad y la mortalidad relacionadas con ella. Pese a todo esto, en los últimos años se pone de manifiesto, a través de un número importante de estudios realizados en distintas ciudades, que, aún por debajo de los niveles de calidad del aire considerados como seguros, los incrementos de los niveles de contaminación atmosférica se asocian con efectos nocivos en la salud.

La interpretación de las reacciones que produce la contaminación atmosférica en la salud humana se basa en estudios toxicológicos y epidemiológicos ${ }^{2}$. En los toxicológicos la concentración, duración y condiciones de la exposición es controlada por el investigador de forma precisa, pero la situación creada es artificial. En los epidemiológicos la observación se realiza en condiciones naturales, pero es muy difícil una vez vertido el contaminante saber quiénes y a qué dosis han estado sometidos. Estos problemas han llevado a diseñar métodos especiales para su estudio. Es el caso de los estudios ecológicos de series temporales. En ellos se analizan las variaciones en el tiempo de la exposición al contaminante y el indicador de salud estudiado en una población.

Una de las ventajas de estos estudios es que, al analizar la misma población en diferentes periodos de tiempo (día a día generalmente), muchas de las variables que pueden actuar como factores de confusión a nivel individual no necesitan ser controladas, ya que se mantienen estables dentro de la misma población.

Una de las limitaciones de los estudios ecológicos es la llamada "falacia ecológica" que se define como el error cometido al trasladar las estimaciones realizadas a nivel poblacional al nivel individual, lo que afecta más a los diseños geográficos que a los de series temporales. También hay que tener en cuenta que, al tratar con mezclas de contaminantes, es complejo atribuir los efectos sobre la salud a uno en concreto.

El proyecto $\mathrm{APHEA}^{3}$ diseñó lo que sería luego un modelo a seguir: el estudio de la evolución en el tiempo de diferentes variables, para luego introducirse en el análisis multivariado, aplicando la regresión de Poisson, relacionando variables respuesta (mortalidad y morbilidad) con variables explicativas (contaminación).

El objetivo del estudio ha consistido en analizar, a corto plazo, la relación existente entre la contaminación atmosférica y los ingresos hospitalarios por enfermedades respiratorias, infecciosas, circulatorias y cardiovasculares, así como la mortalidad en la capital de Albacete durante el año 2005.

\section{MATERIAL Y MÉTODO}

El área geográfica de estudio es la capital de Albacete. Se trata de un estudio transversal de tipo ecológico que asocia dos tipos de variables: ambientales y sanitarias.

Como variables ambientales se recogieron los datos diarios de los contaminantes y variables meteorológicas, calculando el valor promedio diario, el valor máximo diario y mínimo diario de las 24 determinaciones horarias correspondientes a cada día del año 2005 suministrados por Consejería de Medio Ambiente de Castilla la Mancha.

Como variables sanitarias se han utilizado los registros diarios de morbilidad hospitalaria por grupos de edad, sexo y causa (respiratoria, circulatoria, infecciosa y total) que forman parte de la encuesta de morbilidad hospitalaria, y de mortalidad por edad, sexo y todas las causas según datos suministrados por el INE, los cuales derivan de los boletines estadísticos de mortalidad.

Se ha realizado un análisis estadístico con descripción de las distintas variables, ambientales, de morbilidad hospitalaria y de mortalidad, usando las correlacione bivariadas ( $r$ de Pearson) y los modelos de series temporales (ARIMA).

Se han descrito las variaciones temporales, tomando el día como unidad de análisis, de los ingresos hospitalarios por enfermedades respiratorias, infecciosas, circulatorias, cardiovasculares y la mortalidad en la ciudad de Albacete en el año 2005. Igualmente se han descrito las variaciones temporales, tomando el día como unidad de análisis, de los indicadores de contaminación atmosférica y variables meteorológicas durante el año 2005. Esta información ha permitido analizar la relación a corto plazo entre la contaminación atmosférica y los ingresos hospitalarios por dichas enfermedades o la mortalidad.

\section{RESULTADOS}

Las correlaciones bivariadas que se han obtenido entre variables atmosféricas y morbilidad hospitalaria y mortalidad general han sido (tabla 1):

1. Ozono medio. Todos los valores de Ozono medio, máximo y mínimo presentan coeficientes de correlación de Pearson negativos con las variables de morbi-mortalidad, con elevada significación estadís- 
tica. La morbilidad hospitalaria de causa respiratoria en todos los grupos de edad $(r=-0,346, p=0,000)$, en los menores de 14 años $(r=-0,311, p=0,000)$, y en los mayores de 65 años $(r=-0,308, p=0,000)$ se comportan de forma que cuanto más altos son los niveles de ozono menores son las cifras de ingreso por esta patología; la morbilidad global en los menores de 14 años $(r=-0,178, p=0,001)$ y la morbilidad global en los mayores de 65 años $(r=-0,162, p=0,002)$ presentan la misma relación. La mortalidad global en los mayores de 65 años $(r=-0,189, p=0,000)$ y la mortalidad global en todos los grupos de edad $(r=-0,200, p=0,000)$ muestra también la misma relación inversa.

2. Temperatura media. Al igual que ocurre con los niveles de ozono, la temperatura presenta correlaciones inversas, estadísticamente significativas, con los ingresos hospitalarios por patología respiratoria. La morbilidad respiratoria en todos los grupos de edad $(r=-0,446, p=0,000)$, en los menores de 14 años $(r=-0,414, p=0,000)$ y en los mayores de 65 años $(r=-0,392, p=0,000)$ crece cuando la temperatura disminuye. La morbilidad general en los menores de 14 años $(r=-0,192, p=0,000)$ y mayores de 65 años $(r=-0,183, p=0,000)$ se relaciona de la misma forma. La mortalidad global en los mayores de 65 años $(r=-0,189, p=0,000)$ y en el conjunto de todos los grupos de edad $(r=-0,180, p=0,001)$ muestra relaciones semejantes.

3. Radiación solar media. También presenta coeficientes de correlación de Pearson negativos. Son estadísticamente significativas las correlaciones de los ingresos por patología respiratoria en todos los grupos de edad $(r=-0,262, p=0,000)$, con coeficientes más altos en los menores de 14 años $(r=-0,255, p=0,000)$ y en los mayores de 65 años $(r=-0,227, p=0,000)$.

4. Presión barométrica media. Solo los ingresos hospitalarios por patología respiratoria en los mayores de 65 años $(r=0,121, p=0,021)$ y en el conjunto de todos los grupos de edad ( $r=0,117, p=0,026)$, así como la mortalidad general de los mayores de 65 años $(r=0,137, p=0,009)$ y la mortalidad de todos los grupos de edad $(r=0,158, p=0,003)$ muestran correlaciones positivas con los niveles de presión atmosférica.

5. Humedad relativa. Se encuentran coeficientes de correlación positivos entre los niveles medios de hu-

\begin{tabular}{|c|c|c|c|c|c|c|}
\hline EDAD & PM10 & $\mathrm{SO}_{2}$ & $N O_{x}$ & co & $\mathrm{O}_{3}$ & TMP \\
\hline Todas & $\operatorname{MBR}(0,190)$ & $\begin{array}{l}\text { MBG }(0,150) \\
\text { MBR }(0,308) \\
\text { MBC }(0,149) \\
\text { MBI }(0,168) \\
\text { MTG }(-0,182)\end{array}$ & $\begin{array}{l}\text { MBG }(0,210) \\
\text { MBR }(0,389) \\
\text { MBC }(0,235) \\
\text { MTG }(0,251)\end{array}$ & $\begin{array}{l}\text { MBR }(0,263) \\
\text { MTG }(0,216)\end{array}$ & $\begin{array}{l}\text { MBR }(-0,346) \\
\text { MTG }(-0,200)\end{array}$ & $\begin{array}{l}\text { MBR }(-0,446) \\
\text { MTG }(-0,180)\end{array}$ \\
\hline \pm 65 & $\begin{array}{l}\text { MBG }(0,141) \\
\text { MBR }(0,224) \\
\text { MTG }(0,122)\end{array}$ & $\begin{array}{l}\operatorname{MBG}(0,163) \\
\operatorname{MBR}(0,2939)\end{array}$ & $\begin{array}{l}\text { MBG }(0,276) \\
\text { MBR }(0,358) \\
\text { MBC }(0,258) \\
\text { MTG }(0,232)\end{array}$ & $\begin{array}{l}\text { MBR }(0,219) \\
\text { MTG }(0,219)\end{array}$ & $\begin{array}{l}\text { MBR }(-0,308) \\
\text { MTG }(-0,189)\end{array}$ & $\begin{array}{l}\text { MBR }(-0,392) \\
\text { MTG }(-0,189)\end{array}$ \\
\hline $45-64$ & MBG $(0,200)$ & MBG $(0,167)$ & & & & \\
\hline \multicolumn{7}{|l|}{$15-44$} \\
\hline $0-14$ & & $\begin{array}{l}\text { MBG }(0,129) \\
\text { MBR }(0,213)\end{array}$ & $\operatorname{MBR}(0,269)$ & & MBR $(-0,311)$ & MBR $(-0,414)$ \\
\hline
\end{tabular}

Tabla 1. Análisis de correlaciones bivariadas entre variables físico-químicas y variables de morbilidad cuando $p<0,001$. MBG: morbilidad general; MBR: morbilidad por respiratorio; MBC: morbilidad por circulatorio; MBI: morbilidad por infeccioso; MTG: mortalidad general. Entre paréntesis se indica el coeficiente de correlación de Pearson ( $r$ ).

En negrita se indican los coeficientes mayores de 0,3. Edad en años.

\begin{tabular}{l|ccc}
\hline & Modelo & R_estacionaria & NS (p) \\
\hline MBT & ARIMA (0,0,7) & 0,411 & 0,000 \\
MBG $\geq 65$ años & ARIMA $(0,0,14)$ & 0,520 & 0,000 \\
MBG. 45-64 & ARIMA (0,0,14) & 0,457 & 0,000 \\
MBG 15-44 & ARIMA (0,0,14) & 0,212 & 0,000 \\
MBR & ARIMA $(0,0,14)$ & 0,570 & 0,000 \\
MBR $\geq 65$ & ARIMA $(0,0,14)$ & 0,575 & 0,000 \\
MBC & ARIMA (0,0,9) & 0,311 & 0,000 \\
\hline
\end{tabular}

Tabla 2. Modelos de series temporales. Variables independientes: PM10, SO2, NO2, NO, CO, O3 y TMP; Variables dependientes asociadas según modelo ARIMA $(p<0,001)$. 


\begin{tabular}{|c|c|c|c|c|c|}
\hline & Mínimo & Máximo & Media & Val. lím. legal & Desv. típ. \\
\hline PM10-MEDIA $\left(\mu \mathrm{g} / \mathrm{m}^{3}\right)$ & 13,45 & 184,50 & 47,9711 & 150 & 18,28310 \\
\hline PM10-MÁXIMO ( $\left.\mu \mathrm{g} / \mathrm{m}^{3}\right)$ & 16,00 & 437,00 & 87,2865 & & 47,57089 \\
\hline SO2-MEDIA $\left(\mu \mathrm{g} / \mathrm{m}^{3}\right)$ & 2,00 & 22,00 & 4,3702 & 80 & 12,85715 \\
\hline SO2-MÁXIMO $\left(\mu \mathrm{g} / \mathrm{m}^{3}\right)$ & 2,00 & 32,00 & 7,0386 & & 5,09779 \\
\hline NO2-MEDIA $\left(\mu \mathrm{g} / \mathrm{m}^{3}\right)$ & 3,00 & 44,33 & 15,0737 & 200 & 6,84761 \\
\hline NO2-MÁXIMO $\left(\mu \mathrm{g} / \mathrm{m}^{3}\right)$ & 6,00 & 95,00 & 32,8072 & & 16,99273 \\
\hline NO-MEDIA $\left(\mu \mathrm{g} / \mathrm{m}^{3}\right)$ & 2,25 & 57,48 & 8,5938 & 200 & 7,76303 \\
\hline NO-MÁXIMO $\left(\mu \mathrm{g} / \mathrm{m}^{3}\right)$ & 2,00 & 257,00 & 31,8871 & & 39,33783 \\
\hline CO-MEDIA $\left(\mu \mathrm{g} / \mathrm{m}^{3}\right)$ & 0,11 & 1,47 & 0,3654 & 34 & 0,20355 \\
\hline CO-MÁXIMO $\left(\mu \mathrm{g} / \mathrm{m}^{3}\right)$ & 0,13 & 12,40 & 0,7133 & & 0,76644 \\
\hline OZONO-MEDIA $\left(\mu \mathrm{g} / \mathrm{m}^{3}\right)$ & 8,83 & 129,79 & 67,6137 & 180 & 25,69381 \\
\hline OZONO-MÁXIMO $\left(\mu \mathrm{g} / \mathrm{m}^{3}\right)$ & 27,00 & 177,00 & 101,3887 & & 28,94758 \\
\hline TMP-MEDIA $\left({ }^{\circ} \mathrm{C}\right)$ & $-5,31$ & 32,39 & 15,0682 & & 8,64714 \\
\hline TMP-MÁXIMO $\left({ }^{\circ} \mathrm{C}\right)$ & 1,10 & 41,40 & 22,1304 & & 9,54226 \\
\hline TMP-MÍNIMO ( $\left.{ }^{\circ} \mathrm{C}\right)$ & $-11,60$ & 25,30 & 8,8446 & & 7,95202 \\
\hline HR-MEDIA (\%) & 18,00 & 95,42 & 53,7655 & & 17,31334 \\
\hline HR-MÁXIMO (\%) & 23,20 & 99,00 & 77,4235 & & 18,09524 \\
\hline HR-MÍNIMO (\%) & 6,20 & 81,20 & 29,8402 & & 14,67462 \\
\hline PRB-MEDIO (mb) & 920,00 & 953,88 & 937,6471 & & 5,36556 \\
\hline PRB-MÁXIMO (mb) & 889,37 & 956,00 & 939,4473 & & 5,84156 \\
\hline PRB-MÍNIMO (mb) & 919,00 & 953,00 & 935,8953 & & 5,52943 \\
\hline RS-MEDIA (Kwh/m²) & 36,00 & 920,00 & 237,3824 & & 99,57114 \\
\hline RS-MÁX. (Kwh/m²) & 26,00 & 1073,00 & 750,6942 & & 221,81198 \\
\hline
\end{tabular}

Tabla 3. Estadísticos descriptivos de variables contaminantes y atmosféricas en Albacete capital en el año 2005. Notas: PM10: partículas, SO2: dióxido de azufre, NO2: dióxido de nitrógeno, NO: monóxido de nitrógeno, CO: monóxido de carbono, TMP: temperatura, HR: humedad relativa del aire, PRB: presión barométrica, RS: radiación solar.

medad relativa y el número de ingresos hospitalarios por patología respiratoria en los menores de 14 años $(r=0,193, p=0,000)$, los mayores de 65 años $(r=0,113$, $p=0,031)$, los adultos de 15 a 44 años $(r=0,104$, $\mathrm{p}=0,049)$ y la patología respiratoria sin considerar la edad $(r=0,153, p=0,003)$. La humedad relativa máxima ha presentado coeficientes de Pearson positivos con las variables mortalidad total $(r=0,124, p=0,019)$ y mortalidad en mayores de 65 años $(r=0,13, p=0,01)$.

6 . Velocidad del viento. Solo se encuentra una correlación negativa entre la velocidad media del viento y la mortalidad general en el grupo de 45 a 64 años $(r=-0,124, p=0,039)$.

7. Partículas (PM10). Aparecen coeficientes de correlación positivos entre los niveles medios y máximos de partículas y la morbilidad general en adultos de 45 a 64 años $(r=0,120, p=0,020)$, en mayores de 65 años $(r=0,141, p=0,007)$ y en el conjunto de grupos de edad $(r=0,127, p=0,15)$. Los ingresos hospitalarios por causa respiratoria en mayores de 65 años $(r=0,224$, $\mathrm{p}=0,000)$ y en el grupo total por esta patología, con independencia de la edad $(r=0,190, \quad p=0,000)$, se correlacionan igualmente con los niveles de partículas. La mortalidad global de los mayores de 65 años $(r=0,122, p=0,020)$ y la de todos los grupos de edad $(r=0,118, p=0,024)$ muestran coeficientes de Pearson significativos.

8. Dióxido de azufre. Los niveles de $\mathrm{SO}_{2}$ medios y máximos se correlacionan positivamente con la morbilidad hospitalaria general (todas las causas, todas las edades) de manera estadísticamente significativa $(r=0,156, p=0,003)$, en los menores de 14 años $(r=0,129, p=0,014)$, en los de 45-64 años $(r=0,167$, $p=0,001)$ y en los mayores de 65 años $(r=0,163$, $\mathrm{p}=0,002)$, así como con la mortalidad general $(0,182$, $\mathrm{p}=0,000)$. El mejor coeficiente de correlación del $\mathrm{SO}_{2}$ medio y máximo lo presenta la patología respiratoria en el conjunto de ingresos hospitalarios de todas las edades $(r=0,308, p=0,000)$ e igualmente en los menores de 14 años $(r=0,213, p=0,000)$ y en los mayores de 65 años $(r=0,293, p=0,000)$. También se correlacionan significativamente el $\mathrm{SO}_{2}$ máximo y los ingresos por causa de patología del aparato circulatorio en el conjunto de todas las edades $(r=0,149, p=0,004)$ y los de causa infecciosa $(r=0,168 p=0,001)$.

9. Dióxido de nitrógeno. Los niveles de $\mathrm{NO}_{2}$ medio se correlacionan con la morbilidad general $(r=0,210$, $\mathrm{p}=0,000)$, sobretodo en los mayores de 65 años $(0,276, p=0,000)$, y con la mortalidad general $(r=0,251$, $\mathrm{p}=0,000)$ también en los mayores de 65 años $(r=0,232$, $\mathrm{p}=0,000)$. El coeficiente más alto se encuentra entre el $\mathrm{NO}_{2}$ medio y el conjunto de ingresos por patología respiratoria $(r=0,389, p=0,000)$, siendo también significativo en todos los intervalos de edad: menores de 14 años $(r=0,269, p=0,000)$, de 15 a 44 años $(0,153$, $p=0,004)$, de 45 a 64 años $(r=0,254, p=0,000)$ y de 65 y más años $(r=0,358, p=0,000)$. Los ingresos por patología del aparato circulatorio se correlacionan igualmente con los niveles de $\mathrm{NO}_{2}$, tanto en el conjunto de los grupos de edad $(r=0,235, p=0,000)$ como en los mayores de 65 años $(r=0,258, p=0,000)$. Los ingresos 


\begin{tabular}{|c|c|c|c|c|c|}
\hline & Edad (años) & Mínimo & Máximo & Media & Desviación típica \\
\hline $\begin{array}{l}\text { Morbilidad } \\
\text { General }^{1}\end{array}$ & $\begin{array}{c}0-14 \\
15-44 \\
45-64 \\
65 \text { y mas } \\
\text { todos }\end{array}$ & $\begin{array}{c}0 \\
8 \\
1 \\
1 \\
19\end{array}$ & $\begin{array}{c}19 \\
57 \\
4 \\
97 \\
194\end{array}$ & $\begin{array}{c}7,4932 \\
3,463 \\
17,356 \\
38,5342 \\
93,5233\end{array}$ & $\begin{array}{c}4,25145 \\
1,421 \\
8,5877 \\
19,89373 \\
37,13389\end{array}$ \\
\hline Infeccioso $^{1}$ & $\begin{array}{c}0-14 \\
15-44 \\
45-64 \\
65 \text { y mas } \\
\text { todos } \\
5\end{array}$ & $\begin{array}{l}0 \\
0 \\
0 \\
0 \\
0\end{array}$ & $\begin{array}{l}5 \\
5 \\
3 \\
5 \\
8\end{array}$ & $\begin{array}{l}0,8356 \\
0,3562 \\
0,2137 \\
0,4466 \\
1,8521\end{array}$ & $\begin{array}{c}0,97797 \\
0,637 \\
0,46684 \\
0,7491 \\
1,64251\end{array}$ \\
\hline Circulatorio $^{1}$ & $\begin{array}{c}0-14 \\
15-44 \\
45-6415-44 \\
65 \text { y mas } \\
\text { todos }\end{array}$ & $\begin{array}{l}0 \\
0 \\
0 \\
0 \\
0\end{array}$ & $\begin{array}{c}4 \\
5 \\
9 \\
24 \\
3\end{array}$ & $\begin{array}{c}0,63 \\
0,8164 \\
2,6986 \\
9,526 \\
13,141\end{array}$ & $\begin{array}{c}0,32119 \\
1,4395 \\
2,488 \\
5,8359 \\
7,5462\end{array}$ \\
\hline Respiratorio $^{1}$ & $\begin{array}{c}0-14 \\
15-44 \\
45-64 \\
65 \text { y mas } \\
\text { todos }\end{array}$ & $\begin{array}{l}0 \\
0 \\
0 \\
0 \\
0\end{array}$ & $\begin{array}{c}1 \\
7 \\
9 \\
42 \\
55\end{array}$ & $\begin{array}{c}1,5726 \\
1,188 \\
1,326 \\
7,11 \\
11,94\end{array}$ & $\begin{array}{c}1,75515 \\
1,34661 \\
1,5444 \\
6,7819 \\
8,93936\end{array}$ \\
\hline $\begin{array}{l}\text { Mortalidad } \\
\text { General }^{2}\end{array}$ & $\begin{array}{c}0-14 \\
15-44 \\
45-64 \\
65 \text { y mas } \\
\text { todos }\end{array}$ & $\begin{array}{l}0 \\
0 \\
0 \\
0 \\
0\end{array}$ & $\begin{array}{c}1 \\
2 \\
3 \\
10 \\
10\end{array}$ & $\begin{array}{c}0,31 \\
0,126 \\
0,4685 \\
2,9123 \\
3,537\end{array}$ & $\begin{array}{c}0,1712 \\
0,3639 \\
0,66895 \\
1,94936 \\
2,1882\end{array}$ \\
\hline
\end{tabular}

Tabla 4. Estadísticos descriptivos de las variables de morbi-mortalidad de Albacete capital en el año $2005 .{ }^{1}$ Ingresos diarios, ${ }^{2}$ Muertes diarias.

\begin{tabular}{|c|c|c|c|c|c|c|c|}
\hline Var. 1 & Var. 2 & CC & N.S. & Var. 1 & Var. 2 & $\mathrm{CC}$ & N.S. \\
\hline \multirow{6}{*}{ PM10 Md } & TMP Mx & 0,347 & 0,000 & \multirow{11}{*}{ NO Md } & $\mathrm{SO}^{2} \mathrm{Mx}$ & 0,359 & 0,000 \\
\hline & HUMR Md & 0,375 & 0,000 & & $\mathrm{NO}^{2} \mathrm{Md}$ & 0,506 & 0,000 \\
\hline & VELOV Mx & 0,376 & 0,000 & & $\mathrm{CO} \mathrm{Md}$ & 0,464 & 0,000 \\
\hline & RSOL Md & 0,304 & 0,000 & & OZONO Md & $-0,695$ & 0,000 \\
\hline & & & & & VELOV Md & $-0,296$ & 0,000 \\
\hline & & & & & TMP Md & $-0,475$ & 0,000 \\
\hline \multirow{5}{*}{ SO2 Md } & & & & & HUMR Md & 0,322 & 0,000 \\
\hline & NO Md & 0,210 & 0,000 & & PRB Md & 0,253 & 0,000 \\
\hline & OZONO Md & $-0,152$ & 0,003 & & RSOL Md & $-0,393$ & 0,000 \\
\hline & TMP Md & $-0,222$ & 0,000 & & & & \\
\hline & PRB Md & $-0,301$ & 0,000 & & & & \\
\hline \multirow[t]{9}{*}{ NO2 Md } & \multirow{9}{*}{$\begin{array}{l}\text { NO Md } \\
\text { CO Md } \\
\text { OZONO Md } \\
\text { VELOV Md } \\
\text { TMP Md } \\
\text { HUMR Md } \\
\text { PRB Md } \\
\text { RSOL Md }\end{array}$} & 0,506 & 0,000 & \multirow[t]{5}{*}{$\mathrm{CO} \mathrm{Md}$} & OZONO Md & $-0,447$ & 0,000 \\
\hline & & 0,509 & 0,000 & & TMP Md & $-0,329$ & 0,000 \\
\hline & & $-0,642$ & 0,000 & & PRB Md & $-0,305$ & 0,000 \\
\hline & & $-0,413$ & 0,000 & & & & \\
\hline & & $\begin{array}{r}-0,459 \\
0 ? 7 ?\end{array}$ & 0,000 & & & & \\
\hline & & 0,430 & 0,000 & \multirow{4}{*}{$\begin{array}{l}\text { OZONO } \\
\text { Md }\end{array}$} & TMP Mx & 0,752 & 0,000 \\
\hline & & $-0,433$ & 0,000 & & HUMR Md & $-0,569$ & 0,000 \\
\hline & & & & & VELOV Md & 0,207 & 0,001 \\
\hline & & & & & PRB Md & 0,197 & 0,000 \\
\hline
\end{tabular}

Tabla 5. Análisis de correlaciones bivariadas entre las variables físico-químicas de la atmósfera. Notas: Var 1 y Var 2: variables 1 y 2, Md: valores medios, Mx valores máximos, N.S.: nivel de significación (p), CC: coeficiente de correlación de Pearson (r), PM10: partículas, SO2: dióxido de azufre, NO2: dióxido de nitrógeno, NO: monóxido de nitrógeno, CO: monóxido de carbono, TMP: temperatura, HUMR: humedad relativa, PRB: presión barométrica, RSOL: radiación solar, VELOV: velocidad del viento. 


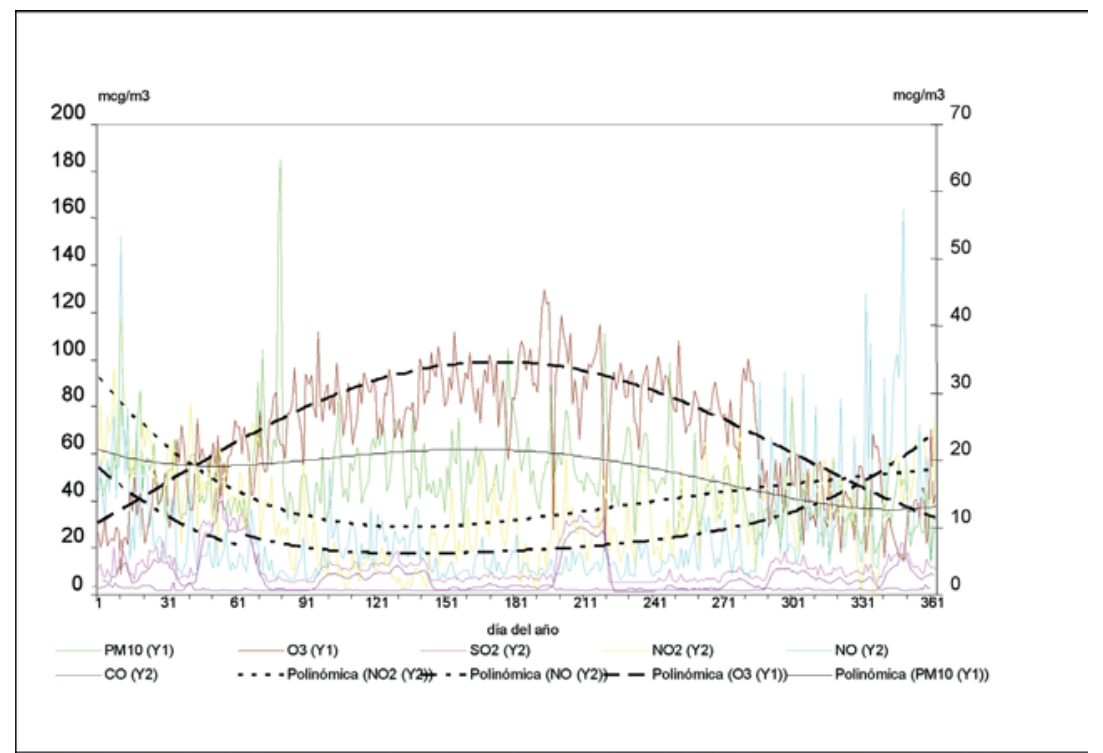

Figura 1. Valores medios diarios y tendencia de la concentración de contaminantes químicos de la atmósfera en la ciudad de Albacete durante 2005

por patología infecciosa y los niveles medios de $\mathrm{NO}_{2}$ se correlacionan de manera estadísticamente significativa y especialmente en los menores de 14 años $(r=0,217, p=0,000)$.

10. Monóxido de nitrógeno. Las correlaciones son semejantes a las encontradas con el dióxido de nitrógeno. Los niveles medios y máximos de NO se correlacionan con la morbilidad general $(r=0,237, p=0,000)$ $y$ en todos los grupos de edad, especialmente en los menores de 14 años $(r=0,211, p=0,000)$ y en los mayores de 65 años $(r=0,241, p=0,000)$. La mortalidad general $(r=0,194, p=0,000)$ y la de los mayores de 65 años $(r=0,186, p=0,000)$ presenta correlaciones significativas con los niveles de NO. La morbilidad hospitalaria por patología respiratoria se correlaciona con los niveles medios de NO ( $r=0,301, p=0,000)$, siendo significativa en todos los grupos de edad, con mejores coeficientes entre los menores de 14 años $(r=0,277$, $p=0,000)$ y en los mayores de 65 años $(r=0,261$, $p=0,000$ ). Los ingresos hospitalarios por patología del aparato circulatorio presentan correlaciones positivas en el grupo formado por todas las edades $(r=0,232$, $p=0,000)$ y en todos los grupos de edad, sobretodo en los mayores de 65 años $(r=0,215, p=0,000)$.

11. Monóxido de carbono. Tan solo los ingresos hospitalarios por patología respiratoria muestran coeficientes de correlación positivos $(r=0,263, p=0,000)$, con mayor coeficiente en los mayores de 65 años $(r=0,276$, $p=0,000)$, y la mortalidad general $(r=0,216, p=0,000)$ y la de mayores de 65 años $(r=0,219 p=0,000)$ se correlacionan con los niveles medios de $\mathrm{CO}$.

Cuando se estudian ${ }^{4}$ en los modelos de series temporales (ARIMA) ${ }^{5}$ (tabla 2) las variables independientes ambientales (PM10, SO2, NO2, NO, CO, O3, TMP) y las variables dependientes sanitarias de morbilidad hospitalaria por diferentes causas y la mortalidad general, se observan los resultados que se detallan a continuación:

- Morbilidad total: el conjunto de las variables independientes contribuye a explicar el $41,1 \%$ de los casos de morbilidad total $(p=0,000)$.

- Morbilidad en 65 y más años: las variables independientes influyen en el $52 \%$ de los casos de la morbilidad en los de 65 y más años $(p=0,000)$.

- Morbilidad en el grupo de edad de 45-64 años: las variables independientes se relacionan con el $45,7 \%$ de los casos de la morbilidad en el grupo de edad de 45-64 $(\mathrm{p}=0,000)$.

- Morbilidad en el grupo de edad de 15-44: las variables atmosféricas independientes influyen en el $21,2 \%$ de los casos de morbilidad en el grupo de edad de 15-44 años $(p=0,000)$.

- Morbilidad total por causa respiratoria: las variables independientes explican el $57 \%$ de los casos de morbilidad total por causa respiratoria $(p=0,001)$.

- Morbilidad por causa respiratoria en 65 y más años: las variables independientes influyen en el $57,5 \%$ de los casos de morbilidad respiratoria en 65 y más años $(\mathrm{p}=0,000)$.

- Morbilidad por causa respiratoria entre el grupo de 0-14 años: las variables independientes predicen un $21 \%$ en la morbilidad por causa respiratoria en el grupo de 0-14 años $(p=0.052)$.

- Morbilidad por causa circulatoria total: las variables independientes contribuyen a explicar el $31,1 \%$ de los casos de la morbilidad por causas circulatorias totales $(p=0,000)$.

- Morbilidad por causas circulatorias en el grupo de 65 y más años: las variables independientes influyen 
en el $34,6 \%$ de los casos de morbilidad por causas respiratorias en el grupo de edad de 65 y más años $(p=0,003)$.

- Morbilidad por causa circulatoria en el grupo de edad de 45-64 años: las variables atmosféricas independientes se relacionan con el $17 \%$ de los casos de morbilidad por causa respiratoria en el grupo de 4564 años $(p=0.033)$.

- Morbilidad por patología infecciosa: sólo se observa relación de las variables independientes sobre la patología infecciosa en el grupo de 0-14 años de edad, con una influencia de las variables independientes de un $10,1 \%$ sobre la morbilidad infecciosa en el grupo de edad de 0-14 años ( $\mathrm{p}=0.015)$.

- Mortalidad total y en los diferentes grupos de edad: no se ha encontrado ninguna relación estadísticamente significativa.

\section{DISCUSIÓN}

Los resultados obtenidos han de ser analizados teniendo en cuenta que la población de Albacete es pequeña con respecto a los estudios que se han llevado a cabo en grandes ciudades. Los niveles de contaminación en la ciudad de Albacete durante el año 2005 son bajos como se puede ver en la tabla 3, estando generalmente por debajo de los límites establecidos en la legislación (debido a las escasas industrias contaminantes, tráfico en relación al tamaño poblacional, etc). Los más cercanos a los valores límite son los de las PM10 debido a que Albacete es árido y tiene unos niveles de partículas cuyo origen es más geológico que antropogénico. Es una ciudad con un clima mediterráneo-continental que da lugar a rangos extremos de temperatura con episodios de altas concentraciones de ozono en verano.

En la figura 1 se recogen los valores medios diarios y la tendencia en las concentraciones de los contaminantes químicos del estudio. En ella se observa cómo la curva de ozono describe máximos en los meses de verano y mínimos en invierno (a su vez está resumida en su forma polinómica trazada con la línea discontinua negra). En lo que se refiere a los niveles de PM10, tienen máximos en enero y marzo, siguiendo también altos, aunque en menor medida, en julio, agosto y septiembre, para descender en el otoño. Los NOx presentan valores altos en invierno y bajos en primavera y verano, como puede observarse en su trazado polinómico. Los SOx tienen valores anárquicos, con algunos picos y mesetas, no observándose ningún tipo de tendencia.

A pesar del bajo nivel de contaminación, se observan relaciones significativas entre algunos contaminantes atmosféricos y la morbilidad hospitalaria y la mortalidad. El ozono tiene una distribución diferente, presentando valores altos en los meses cálidos y bajos en los meses fríos. Los niveles de ozono medidos en
Albacete superan en verano a veces las concentraciones máximas permitidas. Estos resultados son semejantes a los obtenidos en ciudades de características parecidas.

El proyecto "Air Pollution and Health: a European Approach" (APHEA) (Katsouyanni, 1997) presentó resultados similares en cuanto a la estacionalidad de los contaminantes. El estudio "Metanalisi italiana degli studi effetti a breve termine dell'inquinamento atmosférico" (MISA ${ }^{6}$ ) (Biggeri, 2001) detectó una disminución en la primera mitad de los años 90 de la contaminación atmosférica por monóxido de carbono que fue atribuida a la modernización del parque automovilístico por el uso de catalizadores, seguida de una disminución menos marcada, a partir de 1997, producida sobre todo en ciudades pequeñas. Un fenómeno parecido se observó para el dióxido de azufre, atribuido a causas más variadas, como es la metanización de calefacciones domésticas. Las disminuciones para el dióxido de nitrógeno y las partículas en suspensión fueron más débiles. El ozono se muestró estable o con tendencia al aumento en las grandes ciudades. El efecto estacional les hizo dividir el estudio de la asociación entre contaminación atmosférica y mortalidad en dos partes, según se tratase de la estación fría o cálida.

Los niveles medios de contaminantes obtenidos en este estudio son menores que los obtenidos en los estudios antes mencionados, referidos a grandes núcleos de población. Tan solo en el proyecto "Estudio español sobre la relación entre la contaminación atmosférica y la mortalidad "(EMECAM") (Ballester, 1999), algunas de las ciudades participantes (Vitoria, Zaragoza, Sevilla, Huelva y Castellón) presentan cifras semejantes. Este mismo estudio remarca la estacionalidad de los contaminantes, más acusada en las ciudades del norte de España que en las del sur, debido posiblemente al uso de las calefacciones.

En la tabla 4 se recogen los datos de morbi-mortalidad en Albacete capital en el año 2005: aquí puede verse cómo la morbilidad hospitalaria total es de 93 personas por día, siendo de 2 por causa infecciosa, 13 por causa circulatoria y 11 por motivos respiratorios. El número de muertes diarias se sitúa en un promedio de 3, estando dentro de lo esperado para el número de habitantes de la ciudad.

Los análisis de series temporales (tabla 2) muestran una implicación significativa de los factores ambientales en la morbilidad por todas las causas, y en particular por causas de tipo respiratorio y por patologías de origen circulatorio. El efecto de la contaminación atmosférica es superior en personas mayores de sesenta y cuatro años y en los menores de quince años.

El caso de la contaminación por ozono merece un análisis independiente. Es un contaminante secunda- 
rio generado a partir de otros contaminantes previos, haciéndolo depender de otros factores como la radiación solar, que a su vez depende del número de horas de luz y de la temperatura, por lo que sus niveles son más altos en verano. Es igualmente en verano cuando la morbi-mortalidad es menor, coincidiendo con bajos niveles de ozono. En nuestro estudio la relación de este contaminante con la morbi-mortalidad da como resultado correlaciones negativas, lo que parece atribuirle un efecto protector. Esto es debido a que el periodo de estudio comprende sólo un año, debiendo ampliarse a un número mayor con el fin de valorar mejor el efecto.

No parece clara la existencia de un umbral a partir del cual la contaminación resulta inocua. También deben ser consideradas las limitaciones de la epidemiología para establecer valores estándar, como resume BaIlester ${ }^{8}$ (1995): "los efectos de los riesgos ambientales a bajas dosis son pequeños y su ubicuidad dificulta la comparación entre poblaciones; la medida de la exposición es poco válida, los contaminantes evaluados no son todos los existentes y pueden no ser los más perjudiciales en sentido biológico; existe desconocimiento de los grupos de población susceptible, de las interacciones de los contaminantes y de las formas de relación dosis-respuesta, y finalmente no hay un acuerdo acerca de si los efectos se deben al propio agente evaluado o a derivados o mezclas químicas de contaminantes primarios".

\section{BIBLIOGRAFÍA}

1. Firket M. Fof along the Meuse Valley. Transactions of the Faraday Society 1936; 32:1192-7.

2. A Committe of the environmental and Ocupational Health Assembly of the American Thoracic Society. Health effects of outdoor air pollution. Am J Respir Crit Care Med 1996; 153:3-50.

3. Katsouyanni K, Touloumi G, Samoli E, et al. Confounding and effect modification in the short-term effects of ambient particles on total mortality: results from 29 European cities within the APHEA project. Epidemiology 2001; 12:521-31.

4. Lagorio S. Problemi interpretatvi delle analisi di serie temporali su inquinamieno atmosférico e salute. Epidemiol Prev 2001; 25:18190.

5. Murillo C. Métodos Estadísticos de Series Temporales. Barcelona: SG Editores; 1994.

6. Biggeri A, Bellini P, Terracini B et al. MISA: Metanalisi italiana degli studi sugli effettia breve termine dell'inquinamento atmosférico. Epidemiol Prev 2001; 25 (2) suppl: 1-72.

7. Ballester F, Sáez M, Tenías JM, Pérez-Hoyos S, Daponte A, Bellido $\mathrm{J}$ et al. El proyecto EMECAM: discusión de los resultados en las ciudades participantes. Rev Esp Salud Pública 1999; 72:303-14.

8. Ballester F. Contaminación atmosférica, temperatura y mortalidad: estudio en la ciudad de Valencia. Alacant: Universidad d'Alacant, Departamento de Salut Pública; 1995.

\section{Otra bibliografía consultada:}

- Benavides FG, Segura A, Godoy C. Estadísticas mortalidad en España: pequeños problemas, grandes perspectivas. Revisiones de Salud Pública 1991; 2:43-66.

- Biggeri A, Bellini P, Terracini B et al. MISA: Metanalisi italiana degli studi sugli effettia breve termine dell'inquinamento atmosférico. Epidemiol Prev 2001; 25(2)suppl:1-72.
- Boletín Oficial del Estado 1972. Ley 38/1971, de 22 de diciembre de Protección del Medio Ambiente Atmosférico. BOE $n^{\circ}$ 26/12/72.

- Boletín Oficial del Estado 2002. Ley 1073/2002, de 18 de octubre sobre evaluación y gestión de la calidad del aire ambiente en relación con el dióxido de azufre, dióxido de nitrógeno, óxidos de nitrógeno, partículas, plomo, benceno y monóxido de carbono, BOE $n^{\circ} 260,30 / 10 / 2002$.

- Comisión de las Comunidades Europeas 1998. Propuesta de Directiva del Consejo relativa a los valores límite de dióxido de azufre, óxidos de nitrógeno, partículas y plomo en el aire ambiente. Bruselas: Comisión de las Comunidades Europeas; 1998.

- Diario Oficial de las Comunidades Europeas. Directiva del Consejo de 15 de julio de 1980, relativa a los valores límites y a los valores guía de calidad atmosférica para anhídrido sulfuroso y las partículas en suspensión (80/779/CEE).

- Diario Oficial de las Comunidades Europeas. Directiva del Consejo de 22 de abril de 1999, relativa a los valores límites y a los valores guía de calidad atmosférica para el dióxido de azufre, el dióxido de nitrógeno, el monóxido de nitrógeno, las partículas y el plomo. Diario Oficial no L 163/41 del 29 de junio de 1999 (1999/30/CEE).

- Diario Oficial de las Comunidades Europeas. Directiva del Consejo de 21 de julio de 1989 por la que se modifica la Directiva 80/779/ CEE (89/427/CEE).

- Dockery DW, Pope AC, Xu X et al. An association between air pollution and mortality in six US cities. N Engl J Med 1993; 329:1753-59.

- El proyecto EMECAM. Número monográfico. Rev Esp Salud Pública 1999; 73:267-273.

- Forastiere F, Perucci CA, Arcá M et al. Indirect Estimates of Lung Cancer Death Rates in Italy not Atributable to Active Smoking. Epidemiology 1993; 4:502-10.

- Hatzakis A, Katsouyanni K, Kalandidi A et al. Short-term effects of air pollution on mortality in Athens. Int J Epidemiol 1986; 15:397-9

- Hollan WW, Bennett AE, Cameron IR, Florey CV, Leeder SR, Schilling RS et al. Heath effects of particulate pollution: reappraising the evidence. Am J Epidemiol 1979; 110:525-659.

- Katsouyanni K, Karakatsani A, Messari I, et al. Air pollution ans cause specific mortality in Athens. J Epidemiol Community Health 1990; 44:321-4.

- Levy JI, Chemerynski SM, Sarnat JA. Ozone exposure and mortality:an empiric Bayes metaregression analysis. Epidemiology $2005 ; 16(4): 458-68$.

- Luttmann-Gibson H, Suh HH, Coull BA, Dockery DW, Sarnat SE, Schwartz $\mathrm{J}$ et al. Short-term effects of air pollution on heart rate variability in senior adults in Steubenville, Ohio. J Occup Environ Med 2006; 48(8):780-8.

- Pérez Hoyos S, Sáez M, Barceló M et al. Protocolo EMECAM: Análisis de efecto a corto plazo de la contaminación atmosférica sobre la mortalidad. Rev Esp Salud Pública 1999; 72:177-85

- Pope CA, Thun MJ, Namboodiri MM et al. Particulate air pollution as predictor of mortality in a prospective study of US adults 1995; 151:669-74.

- Sáez M, Pérez Hoyos S, Tobias A et al. Métodos de series temporales en los estudios epidemiológicos sobre contaminación atmosférica. Rev Esp salud Pública 1999; 73:133-43.

- Samet JM. Particulate air pollution and mortality: the Philadelphia story. Epidemiology 1995; 6:471-3.

- Sarnat JA, Brown KW, Schwartz J, coull B, Koutrakis P. Ambient gas concentrations and personal particulate matter exposures: implications for studying the health effects of particles. Epidemiology 2005; 16:385-95.

- Schwartz J. Acute effects of ozone on mortality from the "Air pollution and Health: A European Approach"project. Am J Respir Crit Care Med 2004; 170:1080-7. 